\title{
Refinement on Poisson approximation of CDOs
}

\author{
Nat Yonghint ${ }^{\mathrm{a}}$, Kritsana Neammanee $\mathrm{a}^{\mathrm{a}, \mathrm{b}, *}$ \\ a Department of Mathematics and Computer Science, Faculty of Science, Chulalongkorn University, \\ Bangkok 10330 Thailand \\ b Centre of Excellence in Mathematics, Commission on Higher Education, Bangkok 10400 Thailand
}

*Corresponding author, e-mail: Kritsana.N@chula.ac.th

Received 22 Jun 2020

Accepted 24 Mar 2021

ABSTRACT: A collateralized debt obligation (CDO) is a type of structured asset-backed securities. It is one of the causes of the financial crisis in 2007-2008. Jiao and Karoui [Finance Stoch 13 (2009):151-180] and Neammanee and Yonghint [Bull Malays Math Sci Soc 43 (2020):1135-1152] approximated the mean of CDO tranche loss by using Poisson distribution. In this paper, we improve their results by adding a correction term and show that the order of our bounds is better than those previous results. Our main tools are Stein-Chen's method and the technique from Neammanee and Thongtha [Stoch Model Appl 9 (2006):13-23].

KEYWORDS: CDO tranche pricing, Poisson approximation, Stein-Chen's method

MSC2010: 60F05

\section{INTRODUCTION}

In general, a collateralized debt obligation (CDO) is a security that repackages assets into a product that can be chopped into pieces and then sold on the market. It is called collateralized because the repackaged assets are mortgages, corporate debts, auto loans, or credit card debts which serve as collateral for investors. The CDO is sliced into tranches based on the credit rating of each asset. In the standard $\mathrm{CDO}$ tranche with $n$ underlying portfolios, the $i$-th portfolio is assumed to have a recovery rate $R_{i}>0$. We recall that the recovery rate is the proportion of recoverable bad debts. Then, the percentage loss of a CDO at time $T$ is defined as the total loss on every portfolio:

$$
L(T)=\sum_{i=1}^{n} \frac{\left(1-R_{i}\right)}{n} \mathbb{I}_{\left\{\tau_{i} \leqslant T\right\}},
$$

where $\tau_{i}$ is the default time of the $i$-th portfolio and

$$
\mathbb{I}_{\left\{\tau_{i} \leqslant T\right\}}(x)= \begin{cases}1 & \text { if } \tau_{i}(x) \leqslant T \\ 0 & \text { otherwise }\end{cases}
$$

The Hamberger crisis originated in 2007 from a crisis in the subprime mortgage market in the United States. The default of CDOs was one cause of this crisis. A number of subprime mortgages were packed into CDOs which were traded in the market.
Since each CDO tranche has its own percentage loss, an investor would want to know the percentage loss of each tranche. Then, an important value that we need to calculate is $\mathbb{E}\left[\left(L(T)-z^{*}\right)^{+}\right]$where $(x)^{+}:=\max \{x, 0\}$ is a call function, $z^{*}$ is the attachment point (a limit below which the tranche bears none of the loss) or the detachment point (a limit above which the tranche loss does not increase) of the tranche [1-4]. In this article, we assume that the recovery rate for each of these credits is constant and equal to a fixed value $R>0$. Then

$\mathbb{E}\left[\left(L(T)-z^{*}\right)^{+}\right]=\frac{1-R}{n} \mathbb{E}\left[\left(\sum_{i=1}^{n} \mathbb{I}_{\left\{\tau_{i} \leqslant T\right\}}-\frac{n z^{*}}{1-R}\right)^{+}\right]$.

Thus, our problem is to find the expectation of a call function of sums of the Bernoulli random variables.

Let $Y_{1}, Y_{2}, \ldots, Y_{n}$ be independent Bernoulli random variables with $\mathbb{P}\left(Y_{i}=1\right)=p_{i}=1-\mathbb{P}\left(Y_{i}=0\right)$ and $V_{n}=\sum_{i=1}^{n} Y_{i}$. For small $p_{i}$ 's, we approximate $\mathbb{E}\left[\left(V_{n}-z^{*}\right)^{+}\right]$by the expectation of a Poisson random variable. Let $\mathscr{P}_{\lambda}$ be a Poisson random variable with parameter $\lambda=p_{1}+p_{2}+\cdots+p_{n}$, i.e.

$$
\mathbb{P}\left(\mathscr{P}_{\lambda}=m\right)=\frac{\lambda^{m} \mathrm{e}^{-\lambda}}{m !}, \quad m=0,1, \ldots
$$

In 2009, Jiao and Karoui [3] found a uniform bound on a Poisson approximation of $\mathbb{E}\left[\left(V_{n}-z\right)^{+}\right]$ by the Stein-Chen's method [5]. They approximated 
$\mathbb{E}\left[\left(V_{n}-z\right)^{+}\right]$by $\mathbb{E}\left[\left(\mathscr{P}_{\lambda}-z\right)^{+}\right]-C_{h_{z}}^{\mathscr{P}}$ where $C_{h_{z}}^{\mathscr{P}}=$ $\frac{\operatorname{Var}\left(V_{n}\right)-\lambda}{2(z-1) !} \mathrm{e}^{-\lambda} \lambda^{z-1}$ and $z \in \mathbb{N}$. The result states as

$$
\left|\mathbb{E}\left[\left(V_{n}-z\right)^{+}\right]-\mathbb{E}\left[\left(\mathscr{P}_{\lambda}-z\right)^{+}\right]+C_{h_{z}}^{\mathscr{P}}\right| \leqslant A_{\lambda} \epsilon_{n},
$$

where $\epsilon_{n}=4 \sum_{i=1}^{n} p_{i}^{2}+2\left(\sum_{i=1}^{n} p_{i}^{3}\left(1-p_{i}\right)\right)^{\frac{1}{2}}+$ $12\left(\sum_{i=1}^{n} p_{i}^{2}\right)^{2}$ and $A_{\lambda}$ is a constant which depends on $\lambda$. In 2020, Neammanee and Yonghint [6] showed that

$$
\sup _{z \geqslant 0}\left|\mathbb{E}\left[\left(V_{n}-z\right)^{+}\right]-\mathbb{E}\left[\left(\mathscr{P}_{\lambda}-z\right)^{+}\right]\right| \leqslant\left(2 \mathrm{e}^{\lambda}-1\right) \sum_{i=1}^{n} p_{i}^{2} .
$$

It is easy to see that, the order of $\sum_{i=1}^{n} p_{i}^{2}$ in (2) is sharper than the order of $\left(\sum_{i=1}^{n} p_{i}^{3}\left(1-p_{i}\right)\right)^{\frac{1}{2}}$ in (1). Then the correction term $C_{h_{z}}$ is not effective to reduce the order of the bound in the approximation.

Generally, adding correction terms to an approximation of $\mathbb{E}\left[\left(V_{n}-z\right)^{+}\right]$reduces the order of the bound. Hence, the correction term $C_{h_{z}}^{\mathscr{P}}$ is not suitable for this approximation. In this paper, we choose new correction terms to improve the result of Jiao and Karoui [3] and show that the convergence rate is better than that of Neammanee and Yonghint [6]. We use techniques from Stein[7] and Neammanee and Thongtha [8]. The following theorem is our main result.

Theorem 1 Adopting notation defined above, we have

$$
\begin{aligned}
& \sup _{z \geqslant 0}\left|\mathbb{E}\left[\left(V_{n}-z\right)^{+}\right]-\mathbb{E}\left[\left(\mathscr{P}_{\lambda}-z\right)^{+}\right]-C_{\text {call }}\right| \\
& \leqslant \frac{2\left(2 \mathrm{e}^{\lambda}-1\right)}{|\lambda-1| \vee 1}\left(\sum_{j=1}^{n} p_{j}^{2}\right)^{2}, \text { and } \\
& \left|\mathbb{E}\left[\left(V_{n}-z\right)^{+}\right]-\mathbb{E}\left[\left(\mathscr{P}_{\lambda}-z\right)^{+}\right]-C_{\text {call }}\right| \\
& \leqslant \frac{2\left(2 \mathrm{e}^{\lambda}-1\right)(1+\lambda)}{z(|\lambda-1| \vee 1)}\left(\sum_{j=1}^{n} p_{j}^{2}\right)^{2}
\end{aligned}
$$

for $z \geqslant 1$, where $a \vee b=\max \{a, b\}$ and

$$
\begin{aligned}
C_{\text {call }} & =\sum_{j=1}^{n}\left[\mathbb{E}\left[\left(\mathscr{P}_{\lambda-p_{j}}-z\right)^{+}\right]-\mathbb{E}\left[\left(\mathscr{P}_{\lambda}-z\right)^{+}\right]\right] \\
- & \sum_{j=1}^{n} p_{j}\left[\mathbb{E}\left[\left(\mathscr{P}_{\lambda-p_{j}}-z\right)^{+}\right]-\mathbb{E}\left[\left(\mathscr{P}_{\lambda-p_{j}}+1-z\right)^{+}\right]\right] .
\end{aligned}
$$

Remark 1 (i) From (2), the bound tends to 0, where $\sum_{j=1}^{n} p_{j}^{2} \rightarrow 0$ as $n \rightarrow \infty$. In this case, the rate $\left(\sum_{j=1}^{n} p_{j}^{2}\right)^{2}$ in Theorem 1 is better than the rate $\sum_{j=1}^{n} p_{j}^{2}$ in (2). Hence, the correction term $C_{\text {call }}$ is effective in reducing the order of the bound.

(ii) In the case that $Y_{i}$ 's are identically distributed with $p_{i}=1 / n^{\delta}$ where $\delta>1$, the order of the bound in (2) and Theorem 1 are $O\left(1 / n^{2 \delta-1}\right)$ and $O\left(1 / n^{2(2 \delta-1)}\right)$, respectively. Then the convergence rate of the bound in Theorem 1 is better than that in (2).

\section{PROOF OF Theorem 1}

By Stein-Chen's method, a Stein's equation for Poisson distribution is

$v f(v)-\lambda f(v+1)=h(v)-\mathscr{P}_{\lambda}(h), \quad v=0,1,2, \ldots$,

where $f$ and $h$ are real-valued functions on $\mathbb{N} \cup\{0\}$, and $\mathscr{P}_{\lambda}(h)=\mathbb{E}\left[h\left(\mathscr{P}_{\lambda}\right)\right]$. From the equation, Stein [7] (page 86) obtained

$$
\mathbb{E}\left[h\left(V_{n}\right)\right]=\mathscr{P}_{\lambda}(h)+\sum_{j=1}^{n} p_{j}^{2} \mathbb{E}\left[\Delta g_{\lambda}(h)\left(V_{n}^{(j)}\right)\right],
$$

where $\Delta g_{\lambda}(h)(v)=g_{\lambda}(h)(v+2)-g_{\lambda}(h)(v+1)$, $V_{n}^{(j)}=V_{n}-Y_{j}$, and

$$
\begin{aligned}
& g_{\lambda}(h)(v) \\
& = \begin{cases}0, & v=0, \\
\frac{(v-1) !}{\lambda^{v}} \sum_{i=0}^{v-1} \frac{\lambda^{i}}{i !}\left(h(i)-\mathscr{P}_{\lambda}(h)\right), & v=1,2, \ldots .\end{cases}
\end{aligned}
$$

Let $h$ be a call function, i.e. $h(v)=h_{z}(v)=(v-z)^{+}$ for some $z \geqslant 0$. Then by (3),

$$
\begin{aligned}
\mathbb{E}\left[\left(V_{n}-z\right)^{+}\right]-\mathbb{E}\left[\left(\mathscr{P}_{\lambda}-z\right)^{+}\right] \\
=\sum_{j=1}^{n} p_{j}^{2} \mathbb{E}\left[\Delta g_{\lambda}\left(h_{z}\right)\left(V_{n}^{(j)}\right)\right] .
\end{aligned}
$$

We apply (3) with $h=\Delta g_{\lambda}\left(h_{z}\right)$ and $V_{n}=V_{n}^{(j)}$. Then

$$
\begin{aligned}
\mathbb{E}\left[\Delta g_{\lambda}\left(h_{z}\right)\left(V_{n}^{(j)}\right)\right]=\mathscr{P}_{\lambda-p_{j}}\left(\Delta g_{\lambda}\left(h_{z}\right)\right) \\
+\sum_{\substack{l=1 \\
l \neq j}}^{n} p_{l}^{2} \mathbb{E}\left[\Delta g_{\lambda-p_{j}}\left(\Delta g_{\lambda}\left(h_{z}\right)\right)\left(V_{n}^{(j, l)}\right)\right],
\end{aligned}
$$


where $V_{n}^{(j, l)}=V_{n}-\left(Y_{j}+Y_{l}\right)$. Hence, by (5) and (6) we have

$$
\begin{aligned}
\left|\mathbb{E}\left[\left(V_{n}-z\right)^{+}\right]-\mathbb{E}\left[\left(\mathscr{P}_{\lambda}-z\right)^{+}\right]-\sum_{j=1}^{n} p_{j}^{2} \mathscr{P}_{\lambda-p_{j}}\left(\Delta g_{\lambda}\left(h_{z}\right)\right)\right| \\
\quad=\left|\sum_{j=1}^{n} p_{j}^{2} \sum_{\substack{l=1 \\
l \neq j}}^{n} p_{l}^{2} \mathbb{E}\left[\Delta g_{\lambda-p_{j}}\left(\Delta g_{\lambda}\left(h_{z}\right)\right)\left(V_{n}^{(j, l)}\right)\right]\right| . \quad \text { (7) }
\end{aligned}
$$

Next, we divide the proof into 2 steps.

Step 1. We will show that

$$
\sum_{j=1}^{n} p_{j}^{2} \mathscr{P}_{\lambda-p_{j}}\left(\Delta g_{\lambda}\left(h_{z}\right)\right)=C_{\text {call }} .
$$

Note by (4) that

$$
\begin{aligned}
\mathscr{P}_{\lambda-p_{j}}( & \left.\Delta g_{\lambda}\left(h_{z}\right)\right)=\mathbb{E}\left[\Delta g_{\lambda}\left(h_{z}\right)\left(\mathscr{P}_{\lambda-p_{j}}\right)\right] \\
= & \mathrm{e}^{-\left(\lambda-p_{j}\right)} \sum_{v=0}^{\infty} \frac{\Delta g_{\lambda}\left(h_{z}\right)(v)\left(\lambda-p_{j}\right)^{v}}{v !} \\
= & \mathrm{e}^{-\left(\lambda-p_{j}\right)}\left[\sum_{v=0}^{\infty} \frac{g_{\lambda}\left(h_{z}\right)(v+2)\left(\lambda-p_{j}\right)^{v}}{v !}\right. \\
& \left.-\sum_{v=0}^{\infty} \frac{g_{\lambda}\left(h_{z}\right)(v+1)\left(\lambda-p_{j}\right)^{v}}{v !}\right] \\
= & \mathrm{e}^{-\left(\lambda-p_{j}\right)}\left[-R_{1}+R_{2}+R_{3}-R_{4}\right]
\end{aligned}
$$

where

$$
\begin{aligned}
& R_{1}=\frac{1}{\lambda} \sum_{v=0}^{\infty} \frac{\left(\lambda-p_{j}\right)^{v}}{\lambda^{v}} \sum_{i=0}^{v} \frac{\lambda^{i}}{i !}(i-z)^{+}, \\
& R_{2}=\frac{1}{\lambda} \sum_{v=0}^{\infty} \frac{\left(\lambda-p_{j}\right)^{v}}{\lambda^{v}} \sum_{i=0}^{v} \frac{\lambda^{i}}{i !} \mathscr{P}_{\lambda}\left(h_{z}\right), \\
& R_{3}=\frac{1}{\lambda^{2}} \sum_{v=0}^{\infty}(v+1) \frac{\left(\lambda-p_{j}\right)^{v}}{\lambda^{v}} \sum_{i=0}^{v+1} \frac{\lambda^{i}}{i !}(i-z)^{+}, \\
& R_{4}=\frac{1}{\lambda^{2}} \sum_{v=0}^{\infty}(v+1) \frac{\left(\lambda-p_{j}\right)^{v}}{\lambda^{v}} \sum_{i=0}^{v+1} \frac{\lambda^{i}}{i !} \mathscr{P}_{\lambda}\left(h_{z}\right) .
\end{aligned}
$$

To compute $R_{1}$ and $R_{2}$, we note that

$$
\begin{gathered}
\frac{p_{j}}{\lambda} \sum_{v=0}^{\infty}\left(1-\frac{p_{j}}{\lambda}\right)^{v} \sum_{i=0}^{v} \frac{\lambda^{i}}{i !} f(i)=\sum_{v=0}^{\infty}\left(1-\frac{p_{j}}{\lambda}\right)^{v} \sum_{i=0}^{v} \frac{\lambda^{i}}{i !} f(i) \\
-\left(1-\frac{p_{j}}{\lambda}\right) \sum_{v=0}^{\infty}\left(1-\frac{p_{j}}{\lambda}\right)^{v} \sum_{i=0}^{v} \frac{\lambda^{i}}{i !} f(i) \\
=\sum_{v=0}^{\infty}\left(1-\frac{p_{j}}{\lambda}\right)^{v} \sum_{i=0}^{v} \frac{\lambda^{i}}{i !} f(i)-\sum_{v=0}^{\infty}\left(1-\frac{p_{j}}{\lambda}\right)^{v+1} \sum_{i=0}^{v} \frac{\lambda^{i}}{i !} f(i)
\end{gathered}
$$

$$
\begin{aligned}
& =f(0)+\sum_{v=1}^{\infty}\left(1-\frac{p_{j}}{\lambda}\right)^{v} \sum_{i=0}^{v} \frac{\lambda^{i}}{i !} f(i) \\
& -\sum_{v=1}^{\infty}\left(1-\frac{p_{j}}{\lambda}\right)^{v} \sum_{i=0}^{\nu-1} \frac{\lambda^{i}}{i !} f(i) \\
& =f(0)+\sum_{v=1}^{\infty}\left(1-\frac{p_{j}}{\lambda}\right)^{v}\left[\sum_{i=0}^{v} \frac{\lambda^{i}}{i !} f(i)-\sum_{i=0}^{v-1} \frac{\lambda^{i}}{i !} f(i)\right] \\
& =f(0)+\sum_{v=1}^{\infty}\left(1-\frac{p_{j}}{\lambda}\right)^{v} \frac{f(v) \lambda^{v}}{v !} \\
& =\sum_{v=0}^{\infty}\left(1-\frac{p_{j}}{\lambda}\right)^{v} \frac{f(v) \lambda^{v}}{v !} \\
& =\sum_{v=0}^{\infty} \frac{\left(\lambda-p_{j}\right)^{v} f(v)}{v !}=\mathrm{e}^{\lambda-p_{j} \mathbb{E}}\left[f\left(\mathscr{P}_{\lambda-p_{j}}\right)\right],
\end{aligned}
$$

where $f$ is a real-valued function and $j=1,2, \ldots, n$. Hence

$$
\frac{1}{\lambda} \sum_{v=0}^{\infty}\left(1-\frac{p_{j}}{\lambda}\right)^{v} \sum_{i=0}^{v} \frac{\lambda^{i}}{i !} f(i)=\frac{\mathrm{e}^{\lambda-p_{j}}}{p_{j}} \mathbb{E}\left[f\left(\mathscr{P}_{\lambda-p_{j}}\right)\right] .
$$

If $f=h_{z}$ in (10), then

$$
R_{1}=\frac{\mathrm{e}^{\lambda-p_{j}}}{p_{j}} \mathbb{E}\left[\left(\mathscr{P}_{\lambda-p_{j}}-z\right)^{+}\right] .
$$

If $f=1$, then

$$
R_{2}=\frac{\mathrm{e}^{\lambda-p_{j}}}{p_{j}} \mathbb{E}\left[\left(\mathscr{P}_{\lambda}-z\right)^{+}\right] .
$$

To compute $R_{3}$ and $R_{4}$, we let $S=$ $\frac{1}{\lambda^{2}} \sum_{v=0}^{\infty} v\left(1-\frac{p_{j}}{\lambda}\right)^{v} \sum_{i=0}^{v} \frac{\lambda^{i}}{i !} f(i)$. Then

$$
\begin{aligned}
p_{j} S= & \lambda S-\left(\lambda-p_{j}\right) S \\
= & \frac{1}{\lambda} \sum_{v=0}^{\infty} v\left(1-\frac{p_{j}}{\lambda}\right)^{v} \sum_{i=0}^{v} \frac{\lambda^{i}}{i !} f(i) \\
& \quad-\frac{1}{\lambda}\left(1-\frac{p_{j}}{\lambda}\right) \sum_{v=0}^{\infty} v\left(1-\frac{p_{j}}{\lambda}\right)^{v} \sum_{i=0}^{v} \frac{\lambda^{i}}{i !} f(i) \\
= & \frac{1}{\lambda} \sum_{v=1}^{\infty} v\left(1-\frac{p_{j}}{\lambda}\right)^{v} \sum_{i=0}^{v} \frac{\lambda^{i}}{i !} f(i) \\
& \quad-\frac{1}{\lambda} \sum_{v=0}^{\infty} v\left(1-\frac{p_{j}}{\lambda}\right)^{v+1} \sum_{i=0}^{v} \frac{\lambda^{i}}{i !} f(i) \\
= & \frac{1}{\lambda} \sum_{v=1}^{\infty} v\left(1-\frac{p_{j}}{\lambda}\right)^{v} \sum_{i=0}^{v} \frac{\lambda^{i}}{i !} f(i) \\
& \quad-\frac{1}{\lambda} \sum_{v=1}^{\infty}(v-1)\left(1-\frac{p_{j}}{\lambda}\right)^{v} \sum_{i=0}^{v-1} \frac{\lambda^{i}}{i !} f(i)
\end{aligned}
$$




$$
\begin{aligned}
= & {\left[\frac{1}{\lambda} \sum_{v=1}^{\infty} v\left(1-\frac{p_{j}}{\lambda}\right)^{v} \sum_{i=0}^{v} \frac{\lambda^{i}}{i !} f(i)\right.} \\
& \left.\quad-\frac{1}{\lambda} \sum_{v=1}^{\infty} v\left(1-\frac{p_{j}}{\lambda}\right)^{v} \sum_{i=0}^{v-1} \frac{\lambda^{i}}{i !} f(i)\right] \\
& +\frac{1}{\lambda} \sum_{v=1}^{\infty}\left(1-\frac{p_{j}}{\lambda}\right)^{v} \sum_{i=0}^{v-1} \frac{\lambda^{i}}{i !} f(i) \\
= & \frac{1}{\lambda} \sum_{v=1}^{\infty} v\left(1-\frac{p_{j}}{\lambda}\right)^{v}\left[\sum_{i=0}^{v} \frac{\lambda^{i}}{i !} f(i)-\sum_{i=0}^{v-1} \frac{\lambda^{i}}{i !} f(i)\right] \\
& +\frac{1}{\lambda} \sum_{v=0}^{\infty}\left(1-\frac{p_{j}}{\lambda}\right)^{v+1} \sum_{i=0}^{v} \frac{\lambda^{i}}{i !} f(i) \\
= & \frac{1}{\lambda} \sum_{v=1}^{\infty} v\left(1-\frac{p_{j}}{\lambda}\right)^{v} \frac{\lambda^{v}}{v !} f(v)+\left(1-\frac{p_{j}}{\lambda}\right) \frac{\mathrm{e}^{\lambda-p_{j}}}{p_{j}} \mathbb{E}\left[f\left(\mathscr{P}_{\lambda-p_{j}}\right)\right] \\
= & \frac{1}{\lambda} \sum_{v=1}^{\infty} \frac{\left(\lambda-p_{j}\right)^{v}}{(v-1) !} f(v)+\mathrm{e}^{\lambda-p_{j}}\left(\frac{1}{p_{j}}-\frac{1}{\lambda}\right) \mathbb{E}\left[f\left(\mathscr{P}_{\lambda-p_{j}}\right)\right] \\
= & \frac{\lambda-p_{j}}{\lambda} \sum_{v=0}^{\infty} \frac{\left(\lambda-p_{j}\right)^{v}}{v !} f(v+1)+\mathrm{e}^{\lambda-p_{j}}\left(\frac{1}{p_{j}}-\frac{1}{\lambda}\right) \mathbb{E}\left[f\left(\mathscr{P}_{\lambda-p_{j}}\right)\right] \\
= & \mathrm{e}^{\lambda-p_{j}}\left(1-\frac{p_{j}}{\lambda}\right) \mathbb{E}\left[f\left(\mathscr{P}_{\lambda-p_{j}}+1\right)\right] \\
& +\mathrm{e}^{\lambda-p_{j}}\left(\frac{1}{p_{j}}-\frac{1}{\lambda}\right) \mathbb{E}\left[f\left(\mathscr{P}_{\lambda-p_{j}}\right)\right],
\end{aligned}
$$

where $f$ is a real-valued function and $j=1,2, \ldots, n$. Hence, we have

$$
\begin{aligned}
S=\mathrm{e}^{\lambda-p_{j}}\left[\left(\frac{1}{p_{j}}-\frac{1}{\lambda}\right) \mathbb{E}\left[f\left(\mathscr{P}_{\lambda-p_{j}}+1\right)\right]\right. & \\
& +\left(\frac{1}{p_{j}^{2}}-\frac{1}{\lambda p_{j}}\right) \mathbb{E}\left[f\left(\mathscr{P}_{\lambda-p_{j}}\right)\right] .
\end{aligned}
$$

From this fact and (10), we have

$$
\begin{aligned}
\frac{1}{\lambda^{2}} \sum_{v=0}^{\infty}(v+1)\left(1-\frac{p_{j}}{\lambda}\right)^{v} \sum_{i=0}^{v+1} \frac{\lambda^{i}}{i !} f(i) \\
=\frac{1}{\lambda^{2}} \sum_{v=0}^{\infty}(v+1)\left(1-\frac{p_{j}}{\lambda}\right)^{v} \sum_{i=0}^{v} \frac{\lambda^{i}}{i !} f(i) \\
\quad+\frac{1}{\lambda^{2}} \sum_{v=0}^{\infty}(v+1)\left(1-\frac{p_{j}}{\lambda}\right)^{v} \frac{\lambda^{v+1}}{(v+1) !} f(v+1) \\
=S+\frac{1}{\lambda^{2}} \sum_{v=0}^{\infty}\left(1-\frac{p_{j}}{\lambda}\right)^{v} \sum_{i=0}^{v} \frac{\lambda^{i}}{i !} f(i) \\
\quad+\frac{1}{\lambda^{2}} \sum_{v=0}^{\infty}(v+1)\left(1-\frac{p_{j}}{\lambda}\right)^{v} \frac{\lambda^{v+1}}{(v+1) !} f(v+1) \\
=S+\frac{1}{\lambda^{2}} \sum_{v=0}^{\infty}\left(1-\frac{p_{j}}{\lambda}\right)^{v} \sum_{i=0}^{v} \frac{\lambda^{i}}{i !} f(i) \\
\quad+\frac{1}{\lambda} \sum_{v=0}^{\infty} \frac{\left(\lambda-p_{j}\right)^{v}}{v !} f(v+1)
\end{aligned}
$$

$$
\begin{aligned}
& =S+\frac{\mathrm{e}^{\lambda-p_{j}}}{\lambda p_{j}} \mathbb{E}\left[f\left(\mathscr{P}_{\lambda-p_{j}}\right)\right]+\frac{\mathrm{e}^{\lambda-p_{j}}}{\lambda} \mathbb{E}\left[f\left(\mathscr{P}_{\lambda-p_{j}}+1\right)\right] \\
& =\mathrm{e}^{\lambda-p_{j}}\left[\frac{\mathbb{E}\left[f\left(\mathscr{P}_{\lambda-p_{j}}+1\right)\right]}{p_{j}}+\frac{\mathbb{E}\left[f\left(\mathscr{P}_{\lambda-p_{j}}\right)\right]}{p_{j}^{2}}\right] .
\end{aligned}
$$

If $f=h_{z}$ in (13), then

$R_{3}=\mathrm{e}^{\lambda-p_{j}}\left[\frac{1}{p_{j}} \mathbb{E}\left[\left(\mathscr{P}_{\lambda-p_{j}}+1-z\right)^{+}\right]+\frac{1}{p_{j}^{2}} \mathbb{E}\left[\left(\mathscr{P}_{\lambda-p_{j}^{-}}-\boldsymbol{z}\right)^{+}\right]\right]$.

If $f=1$ in (13), then

$$
R_{4}=\mathrm{e}^{\lambda-p_{j}}\left(\frac{1}{p_{j}}+\frac{1}{p_{j}^{2}}\right) \mathbb{E}\left[\left(\mathscr{P}_{\lambda}-z\right)^{+}\right] .
$$

Hence, (8) follows from (9), (11), (12), (14), (15).

Step 2. We will show that

$$
\begin{aligned}
\left|\sum_{j=1}^{n} p_{j}^{2} \sum_{\substack{l=1 \\
l \neq j}}^{n} p_{l}^{2} \mathbb{E}\left[\Delta g_{\lambda-p_{j}}\left(\Delta g_{\lambda}\left(h_{z}\right)\left(V_{n}^{(j, l)}\right)\right)\right]\right| \\
\leqslant \frac{2\left(2 \mathrm{e}^{\lambda}-1\right)}{(|\lambda-1| \vee 1)}\left(\sum_{j=1}^{n} p_{j}^{2}\right)^{2}
\end{aligned}
$$

and, for $z>1$,

$$
\begin{aligned}
\left|\sum_{j=1}^{n} p_{j}^{2} \sum_{\substack{l=1 \\
l \neq j}}^{n} p_{l}^{2} \mathbb{E}\left[\Delta g_{\lambda-p_{j}}\left(\Delta g_{\lambda}\left(h_{z}\right)\left(V_{n}^{(j, l)}\right)\right)\right]\right| \\
\leqslant \frac{2\left(2 \mathrm{e}^{\lambda}-1\right)(1+\lambda)}{z(|\lambda-1| \vee 1)}\left(\sum_{j=1}^{n} p_{j}^{2}\right)^{2} .
\end{aligned}
$$

To proof Step 2, we note that $\left\|\Delta g_{\lambda}(h)\right\|_{\infty} \leqslant$ $2\|h\|_{\infty} \min (1 / \lambda, 1)$ where $\|h\|_{\infty}=\sup _{v \in \mathbb{N} \cup\{0\}}|h(v)|$ (Stein [7], p. 89). Then

$$
\begin{aligned}
& \left\|\Delta g_{\lambda-p_{j}}\left(\Delta g_{\lambda}\left(h_{z}\right)\right)\right\|_{\infty} \\
& \quad \leqslant 2\left\|\Delta g_{\lambda}\left(h_{z}\right)\right\|_{\infty} \min \left(\frac{1}{\lambda-p_{j}}, 1\right) .
\end{aligned}
$$

Observe that for $\lambda>1$, we have

$$
\min \left(\frac{1}{\lambda-p_{j}}, 1\right) \leqslant \min \left(\frac{1}{\lambda-1}, 1\right) \leqslant \frac{1}{|\lambda-1| \vee 1},
$$

and for $0<\lambda \leqslant 1$,

$$
\min \left(\frac{1}{\lambda-p_{j}}, 1\right)=1=\frac{1}{|\lambda-1| \vee 1} .
$$

Hence by (16)-(18),

$$
\begin{aligned}
& \mathbb{E}\left[\Delta g_{\lambda-p_{j}}\left(\Delta g_{\lambda}\left(h_{z}\right)\left(V_{n}^{(j, l)}\right)\right)\right] \\
& \leqslant \| \Delta g_{\lambda-p_{j}}\left(\Delta g_{\lambda}\left(h_{z}\right) \|_{\infty} \leqslant \frac{2\left\|\Delta g_{\lambda}\left(h_{z}\right)\right\|_{\infty}}{|\lambda-1| \vee 1} .\right.
\end{aligned}
$$


This implies that

$$
\begin{aligned}
\mid \sum_{j=1}^{n} p_{j}^{2} \sum_{\substack{l=1 \\
l \neq j}}^{n} p_{l}^{2} \mathbb{E}\left[\Delta g_{\lambda-p_{j}}\right. & \left.\left(\Delta g_{\lambda}\left(h_{z}\right)\left(V_{n}^{(j, l)}\right)\right)\right] \mid \\
& \leqslant \frac{2\left\|\Delta g_{\lambda}\left(h_{z}\right)\right\|_{\infty}}{|\lambda-1| \vee 1}\left(\sum_{j=1}^{n} p_{j}^{2}\right)^{2} .
\end{aligned}
$$

From this inequality and the facts that $\left|\Delta g_{\lambda}\left(h_{z}\right)\right| \leqslant$ $2 \mathrm{e}^{\lambda}-1$ and $\left|\Delta g_{\lambda}\left(h_{z}\right)\right| \leqslant \frac{\left(2 \mathrm{e}^{\lambda}-1\right)(1+\lambda)}{z}$ for $z>1$ (Neammanee and Yonghint [6]), we have

$$
\begin{aligned}
\left|\sum_{j=1}^{n} \sum_{\substack{l=1 \\
l \neq j}}^{n} p_{j}^{2} p_{l}^{2} \mathbb{E}\left[\Delta g_{\lambda-p_{j}}\left(\Delta g_{\lambda}\left(h_{z}\right)\left(V_{n}^{(j, l)}\right)\right)\right]\right| & \\
& \leqslant \frac{2\left(2 \mathrm{e}^{\lambda}-1\right)}{|\lambda-1| \vee 1}\left(\sum_{j=1}^{n} p_{j}^{2}\right)^{2},
\end{aligned}
$$

and for $z>1$,

$$
\begin{aligned}
\mid \sum_{j=1}^{n} \sum_{\substack{l=1 \\
l \neq j}}^{n} p_{j}^{2} p_{l}^{2} \mathbb{E}\left[\Delta g_{\lambda-p_{j}}\left(\Delta g_{\lambda}\left(h_{z}\right)\left(V_{n}^{(j, l)}\right)\right)\right] \\
\leqslant \frac{2\left(2 \mathrm{e}^{\lambda}-1\right)(1+\lambda)}{z(|\lambda-1| \vee 1)}\left(\sum_{j=1}^{n} p_{j}^{2}\right)^{2} .
\end{aligned}
$$

Combining Steps 1 and 2, the proof is completed.

\section{REFERENCES}

1. Glasserman P, Suchintabandid S (2007) Correlations for CDO pricing. $J$ Bank Finance 31, 1375-1398.

2. Hull J, White A (2004) Valuation of a CDO and an nth to default CDS without Monte Carlo simulation. $J$ Deriv 12, 8-23.

3. Jiao Y, Karoui NEL (2009) Stein's method and zero bias transformation for $\mathrm{CDO}$ tranche pricing. Finance Stoch 13, 151-180.

4. Jiao Y, Karoui NEL, Kurt D (2008) Gauss and Poisson approximation: Applications to CDOs tranche pricing. $J$ Comput Finance 12, 31-58.

5. Chen LHY (1975) Poisson approximation for dependent trials. Ann Probab 3, 534-545.

6. Neammanee K, Yonghint N (2020) Poisson approximation for call function via Stein-Chen method. Bull Malays Math Sci Soc 43, 1135-1152.

7. Stein C (1986) Approximate Computation of Expectations, IMS, Hayward, CA, USA.

8. Neammanee K, Thongtha P (2006) Refinement on bounds of Poisson approximation. Stoch Model Appl 9 , 13-23. 